\title{
融合全模态自编码器和生成对抗机制的跨模态检索
}

\author{
赵鹏 ${ }^{1,2}$ ，马泰宇 ${ }^{1,2)}$ ，李毅 ${ }^{1,2)}$ ，刘慧婷 1,2$)$ \\ 1) (安徽大学计算智能与信号处理教育部重点实验室 合肥 230601) \\ 2) (安徽大学计算机科学与技术学院 合肥 230601) \\ (zhaopeng_ad@163.com)
}

\begin{abstract}
摘 要: 针对现有基于生成对抗网络的跨模态检索方法不能充分挖掘模态间不变性的问题，提出一种融合全模态自 编码器和生成对抗机制的跨模态检索方法. 引人 2 个并行的全模态自编码器, 将不同模态的样本嵌人公共空间, 每个 全模态自编码器不仅重构出自身模态的特征表示, 而且还重构出跨模态的特征表示. 设计了一个分类器, 预测公共 空间中嵌人特征的类别, 学习并保留样本中的语义判别性. 设计了 3 个判别器, 分别判断输人其中的特征所属的模态 类别, 它们协同工作，充分挖掘模态间的不变性. 以平均精度均值为指标评价跨模态检索的精确度, 在 Pascal Sentence, Wikipedia 和 NUS-WIDE-10k 这 3 个公开数据集上进行实验, 实验结果表明, 与 10 个包括传统方法和深度学习 方法在内的跨模态检索的主流方法进行对比，所提方法在 3 个数据集上的平均精度均值分别至少提高了 $4.8 \%, 1.4 \%$ 和 $1.1 \%$, 证明了所提方法的有效性.
\end{abstract}

关键词：全模态自编码器; 生成对抗网络; 跨模态检索

中图法分类号: TP391.41ＤOI: 10.3724/SP.J.1089.2021.18757

\section{Cross-Modal Retrieval Based on Full-Modal Autoencoder with Generative Adversarial Mechanism}

\author{
Zhao Peng ${ }^{1,2)}$, Ma Taiyu ${ }^{1,2)}$, Li Yi ${ }^{1,2)}$, and Liu Huiting ${ }^{1,2)}$ \\ 1) (Key Laboratory of Intelligent Computing and Signal Processing of Ministry of Education, Anhui University, Hefei 230601) \\ ${ }^{2)}$ (School of Computer Science and Technology, Anhui University, Hefei 230601)
}

\begin{abstract}
Existing cross-modal retrieval methods based on generative adversarial networks can't fully explore the inter-modality invariance. Aiming to solve the problem, a novel cross-modal retrieval method based on full-modal autoencoder with generative adversarial mechanism is proposed. Two parallel full- modal autoencoders are introduced to embed samples of different modalities into a common space. Each full-modal autoencoder not only reconstructs the feature representation of its own modality, but also reconstructs the feature representation of the other modality. A classifier is designed to predict the categories of the embedding features in the common space, which aims to preserve the semantic discriminative information of samples. Three discriminators are designed to determine the modal categories of the input features, respectively, and these three discriminators work cooperatively to fully explore the inter-modality invariance. The mean average precision (mAP) is used to evaluate the accuracy of cross-modal retrieval and extensive experiments are conducted on three public datasets which are Pascal Sentence, Wikipedia and
\end{abstract}

收稿日期：2020-11-04; 修回日期：2021-08-02. 基金项目：国家自然科学基金(61602004); 安徽省高校自然科学研究重点项目 (KJ2018A0013, KJ2020A0041); 安徽省自然科学基金(2108085MF212, 2008085MF219, 1908085MF188); 安徽省重点研究与开发计划 (1804d08020309). 赵鹏(1976一), 女, 博士, 副教授, 硕士生导师, CCF 会员, 主要研究方向为机器学习、图像理解; 马泰宇(1996一), 男, 硕士研究生, 主要研究方向为机器学习、跨模态检索; 李毅(1997一), 男, 硕士研究生, 主要研究方向为机器学习; 刘慧婷 (1978一), 女, 博士, 副教授, 硕士生导师, CCF 会员, 主要研究方向为机器学习. 
NUS-WIDE-10k. Compared to ten state-of-the-art cross-modal retrieval methods including traditional methods and deep learning methods, the mAP of the proposed method on the three datasets improves at least $4.8 \%, 1.4 \%$ and $1.1 \%$ on the three datasets respectively. The experimental results prove the effectiveness of the proposed method.

Key words: full-modal autoencoder; generative adversarial networks; cross-modal retrieval

随着图像、文本、视频和音频等多媒体数据爆 炸式地增长, 单模态检索已经不能满足用户越来 越多样化的检索需求，如用户想通过一幅图像检 索相关的音频剪辑或文字等, 因此跨模态检索逐 渐受到学术界和工业界的广泛关注. 跨模态检索 的目标是在给定一个模态查询实例的情况下，精 确地从其他模态中搜索出与之语义相似的实例. 近几年，虽然跨模态检索研究已经取得了巨大进 展，但是 “模态鸿沟”依然是该领域目前存在的巨 大挑战. 由于不同模态的数据分布和表示方式存 在差异，因此无法直接度量它们之间的相似性.

现有的跨模态检索方法主要通过学习一个公 共空间来缩小模态鸿沟, 将不同模态的数据嵌人 公共空间，缩小该空间中语义相关的数据之间的 距离, 增大语义不相关的数据之间的距离, 使不同 模态的样本可以直接在该公共空间中进行相似性 度量. 现有的跨模态检索方法可以分为 2 类. (1) 传统方法 ${ }^{[1-6]}$. 如典型相关性分析 (canonical correlation analysis, CCA $)^{[1]}$ 通过最大化不同模态样本之 间的相关性来学习映射矩阵, 将不同模态的样本 映射到一个公共空间中; 跨模态因子分析 (crossmodal factor analysis, CFA) ${ }^{[2]}$ 通过最小化不同模态 成对样本之间的弗罗贝尼乌斯范数学习线性映射, 将不同模态的样本映射到一个公共空间中. (2) 基 于深度学习的方法 ${ }^{[7-10]}$. 如跨模态混合迁移网络 (cross-modal hybrid transfer network, CHTN) ${ }^{[8]}$ 将从 $^{\text {从 }}$ 单模态数据中学习到的知识在跨模态数据之间共 享, 以提升跨模态检索的精度; 跨模态相关性学习 (cross-modal correlation learning, CCL) ${ }^{[10]}$ 是两阶段 的学习方法, 第 1 阶段通过联合优化模态内和模态 间的相关性学习各个模态样本的特征表示; 第 2 阶 段采用多任务学习策略, 自适应地平衡模态内语 义类别约束和模态间成对数据相似性约束.

此外, 跨模态检索方法根据在训练过程中是 否使用标签信息分为 2 类. (1) 有监督方法 ${ }^{[11-14]}$. 有监督方法可以有效地利用标签中包含的语义信 息区分不同类别的样本，缩小相同类别样本之间 的距离. 如深度跨模态哈希(deep cross-modal hash- ing, $\mathrm{DCMH})^{[13]}$ 将特征学习和哈希码学习集成到同 一个框架中, 用根据类别标签构建的相似度矩阵指 导跨模态数据相似性的学习; 语义深度跨模态哈希 (semantic deep cross-modal hashing, SDCH) ${ }^{[14]}$ 同时 考虑了模态间和模态内的数据相关性, 用一个双 重语义监督损失指导网络学习哈希码. (2) 无监督 方法 ${ }^{[15-18]}$. 如协同矩阵分解哈希 (collective matrix factorization hashing, $\mathrm{CMFH})^{[16]}$ 在公共的潜在语义 空间中学习不同模态样本的哈希码, 跨模态的样 本可以在公共语义空间中直接进行相似性度量; 深度联合语义重构哈希 (deep joint-semantics reconstructing hashing, DJSRH) ${ }^{[18]}$ 构造了一个联合语 义关系矩阵, 以捕捉跨模态样本的内在语义关系, 学习能获得最大限度地重构出上述内在语义关系 的哈希码.

近几年，生成对抗网络(generative adversarial networks, GAN)发展迅速, 其判别器的特点是学习 具有判别性特征的能力较强, 因此越来越多的工 作将 GAN 应用到跨模态检索中 ${ }^{[19-22]}$. 但现有的基 于 GAN 的跨模态检索方法大多只包含一个判别 器, 不能充分地挖掘模态间的不变性, 限制了跨模 态检索的精度. 为了解决这个问题, 本文提出一个 融合全模态自编码器和生成对抗机制的跨模态检 索方法, 引人 2 个并行的全模态自编码器, 分别将 图像和文本模态的样本嵌人公共空间. 为了在公 共空间中更好地保留语义判别性, 本文在 2 个全模 态自编码器的中间层之间设计了一个分类器, 将 公共空间中的嵌人特征映射到类别标签空间中. 为了更加充分地挖掘模态间的不变性，不同于一 般的基于 GAN 的跨模态检索方法仅设计了一个判 别器, 本文设计了 3 个判别器协同工作, 通过对抗 学习, 使最终学习到的公共空间不仅保留语义判 别性，还保留模态间的不变性.

\section{1 相关工作}

\subsection{GAN}

GAN 自提出以来就受到广泛的关注，在图像 
生成、表示学习和跨模态检索等研究中都获得了较 好的效果. GAN 包括一个生成器 $G$ 和一个判别器 $D$, 其中 $D$ 的目标是学习如何区分一个给定的样本 是真实的样本还是生成的样本, 而 $G$ 的目标是生 成使 $D$ 无法识别出真假的样本. $G$ 和 $D$ 以对抗的方 式进行训练, 不断优化直至达到平衡. GAN 的目标 函数为

$$
\begin{gathered}
\min _{G} \max _{D} V(G, D)=E_{\boldsymbol{x} \sim P_{\text {data }}(\boldsymbol{x})}[\ln D(\boldsymbol{x})]+ \\
E_{\boldsymbol{z} \sim P_{z}(\boldsymbol{z})}[\ln (1-D(G(\boldsymbol{z})))] .
\end{gathered}
$$

其中, $E$ 为分布函数的期望值; $x$ 为真实的样本; $z$ 为 输人的噪声; $P_{\text {data }}(\boldsymbol{x})$ 为真实样本的分布; $P_{z}(z)$ 为噪 声的分布.

近年来，基于 GAN 的跨模态检索方法被陆续 提出. 对抗的跨模态检索 (adversarial cross-modal retrieval, ACMR $)^{[19]}$ 将模态分类器和特征映射器进 行对抗训练, 学习具有模态间不变性和模态内判 别性的公共特征表示. 深度对抗的度量学习(deep adversarial metric learning, DAML) ${ }^{[20]}$ 将不同模态 的样本映射到一个公共非线性子空间中，并使用 模态判别器判断嵌人特征的模态. 模态对抗的混 合迁移网络(modal-adversarial hybrid transfer network, MHTN $)^{[22]}$ 用一个模态共享的知识迁移子网 络, 将知识从单模态的源域迁移到跨模态的目标 域中, 并采用对抗机制对生成模型和模态判别模 型进行训练.

\section{2 自编码器}

自编码器是一种无监督学习算法, 包括编码 器和解码器 2 个部分. 训练完成后的编码器可以获 取样本的潜在特征表示, 通常潜在特征的维度比 输人特征的维度小, 可达到降维的目的. 现有的一 些跨模态检索方法 ${ }^{[15,23-24]}$ 将自编码器作为骨干网 络. 多模态语义自编码器 (multi-modal semantic autoencoder, MMSAE ${ }^{[23]}$ 学习将多模态数据投影到 低维嵌人空间中的映射函数. 模态对抗的语义学 习网络 (modal-adversarial semantic learning network, MASLN $)^{[24]}$ 用条件自编码器实现跨模态的特 征重构, 利用对抗学习机制捕获公共空间中类别 之间的语义相关性和判别性.

\section{2 本文方法}

\section{1 问题定义}

本文的跨模态检索涉及 2 种模态：图像(I)和 文本( $\mathrm{T})$. 用 $O=\left\{\left(\boldsymbol{i}_{p}, \boldsymbol{t}_{p}, \boldsymbol{y}_{p}\right)\right\}_{p=1}^{n}$ 表示数据集, 其中, $\boldsymbol{i}_{p}$ 和 $\boldsymbol{t}_{p}$ 分别表示第 $p$ 幅图像的特征向量和其对应 文本的特征向量, 图像特征和文本特征的维度分 别用 $d_{\mathrm{I}}$ 和 $d_{\mathrm{T}}$ 表示, $\boldsymbol{y}_{p}$ 表示该图像-文本对所属类 别标签的 one-hot 向量, $n$ 代表数据集中样本对的总 数, 训练集中样本对的个数用 $n_{\text {train }}$ 表示, 测试集 中样本对的个数用 $n_{\text {test }}$ 表示, $n=n_{\text {train }}+n_{\text {test }}$. 数据 集包含的类别总数用 $c$ 表示. I2T 表示输人查询图 像, 检索与之语义相似的文本的跨模态检索任务. T2I 表示输人查询文本, 检索与之语义相似的图像 的跨模态检索任务.

\section{2 方法概述}

本文方法的网络结构如图 1所示. 整个网络由 生成模型和判别模型组成. 其中生成模型由图像 全模态自编码器 $G_{\mathrm{I}}$ 、文本全模态自编码器 $G_{\mathrm{T}}$ 和分 类器 $C$ 组成. $G_{\mathrm{I}}$ 由编码器 $G_{\mathrm{II}}$ 和 2 个解码器 $\left(G_{\mathrm{IdI}}\right.$ 和 $\left.G_{\mathrm{IdT}}\right)$ 组成. $G_{\mathrm{T}}$ 由编码器 $G_{\mathrm{Te}}$ 和 2 个解码器 $\left(G_{\mathrm{TdT}}\right.$ 和 $G_{\mathrm{TdI}}$ )组成. 不同于一般的自编码器只重构出自 身模态的特征， $G_{\mathrm{I}}$ 和 $G_{\mathrm{T}}$ 不仅重构出自身模态的特 征, 而且还重构出跨模态的特征. 生成模型的目标 是学习 2 个映射函数, 将不同模态的样本嵌人一个 公共空间。

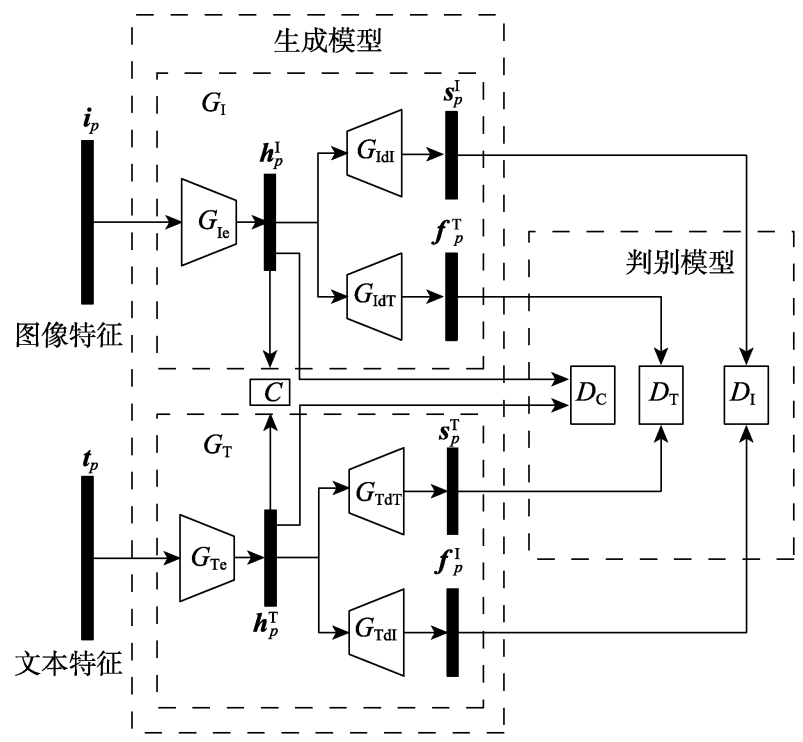

图 1 本文方法的网络结构

判别模型由判别器 $D_{\mathrm{C}}, D_{\mathrm{I}}$ 和 $D_{\mathrm{T}}$ 组成. 判别模 型的目标是充分挖掘模态间的不变性, 并将其保 留在公共空间中.

生成模型和判别模型以对抗的方式协同训练, 最终使学习到的公共空间既具有语义判别性, 又 具有模态间不变性，在该公共空间中能够更好地 度量不同模态的样本之间的相似度. 


\section{3 生成模型}

在训练阶段, $\boldsymbol{i}_{p}$ 通过 $G_{\mathrm{Ie}}$ 得到图像的嵌人特征 $\boldsymbol{h}_{p}^{\mathrm{I}}$, 即 $\boldsymbol{h}_{p}^{\mathrm{I}}=G_{\mathrm{Ie}}\left(\boldsymbol{i}_{p} ; \boldsymbol{\theta}_{\mathrm{Ie}}\right)$, 其中, $\boldsymbol{\theta}_{\mathrm{Ie}}$ 为 $G_{\mathrm{Ie}}$ 的参数. $G_{\mathrm{IdI}}$ 和 $G_{\mathrm{IdT}}$ 分别将 $\boldsymbol{h}_{p}^{\mathrm{I}}$ 重构为图像模态的特征 $\boldsymbol{s}_{p}^{\mathrm{I}}$ 和 文本 模态的特征 $\boldsymbol{f}_{p}^{\mathrm{T}}$, 即 $\boldsymbol{s}_{p}^{\mathrm{I}}=G_{\mathrm{IdI}}\left(\boldsymbol{h}_{p}^{\mathrm{I}} ; \boldsymbol{\theta}_{\mathrm{IdI}}\right)$, $\boldsymbol{f}_{p}^{\mathrm{T}}=G_{\mathrm{IdT}}\left(\boldsymbol{h}_{p}^{\mathrm{I}} ; \boldsymbol{\theta}_{\mathrm{IdT}}\right)$, 其中, $\boldsymbol{\theta}_{\mathrm{IdI}}$ 和 $\boldsymbol{\theta}_{\mathrm{IdT}}$ 分别为 $G_{\mathrm{IdI}}$ 和 $G_{\mathrm{IdT}}$ 的参数. 类似地, $\boldsymbol{t}_{p}$ 通过 $G_{\mathrm{Te}}$ 得到文本的嵌 人特征 $\boldsymbol{h}_{p}^{\mathrm{T}}$, 即 $\boldsymbol{h}_{p}^{\mathrm{T}}=G_{\mathrm{Te}}\left(\boldsymbol{t}_{p} ; \boldsymbol{\theta}_{\mathrm{Te}}\right)$, 其中, $\boldsymbol{\theta}_{\mathrm{Te}}$ 为 $G_{\mathrm{Te}}$ 的参数. $G_{\mathrm{TdI}}$ 和 $G_{\mathrm{TdT}}$ 分别将 $\boldsymbol{h}_{p}^{\mathrm{T}}$ 重构为图像模态的 特 征 $\boldsymbol{f}_{p}^{\mathrm{I}}$ 和 文本 模态的特 征 $\boldsymbol{s}_{p}^{\mathrm{T}}$, 即 $\boldsymbol{f}_{p}^{\mathrm{I}}=$ $G_{\mathrm{TdI}}\left(\boldsymbol{h}_{p}^{\mathrm{T}} ; \boldsymbol{\theta}_{\mathrm{TdI}}\right), \boldsymbol{s}_{p}^{\mathrm{T}}=G_{\mathrm{TdT}}\left(\boldsymbol{h}_{p}^{\mathrm{T}} ; \boldsymbol{\theta}_{\mathrm{TdT}}\right)$; 其中, $\boldsymbol{\theta}_{\mathrm{TdI}}$ 和 $\boldsymbol{\theta}_{\mathrm{TdT}}$ 分别为 $G_{\mathrm{TdI}}$ 和 $G_{\mathrm{TdT}}$ 的参数. 定义重构损失函 数为

$$
\begin{aligned}
& L_{\text {rec }}\left(\boldsymbol{W}_{\text {rec }}\right)=\frac{1}{n_{\text {train }}} \sum_{p=1}^{n_{\text {train }}} l_{\text {rec }}\left(\boldsymbol{i}_{p}, \boldsymbol{t}_{p} ; \boldsymbol{W}_{\text {rec }}\right)= \\
& \frac{1}{n_{\text {train }}} \sum_{p=1}^{n_{\text {train }}}\left(l_{\mathrm{I}}\left(\boldsymbol{i}_{p}, \boldsymbol{t}_{p} ; \boldsymbol{W}_{\text {rec }}\right)+l_{\mathrm{T}}\left(\boldsymbol{i}_{p}, \boldsymbol{t}_{p} ; \boldsymbol{W}_{\text {rec }}\right)\right) .
\end{aligned}
$$

其中, $\boldsymbol{W}_{\text {rec }}$ 表示 $G_{\mathrm{I}}$ 和 $G_{\mathrm{T}}$ 的参数集合, 即 $\boldsymbol{W}_{\text {rec }}=$ $\left[\boldsymbol{\theta}_{\mathrm{Ie}}, \boldsymbol{\theta}_{\mathrm{IdI}}, \boldsymbol{\theta}_{\mathrm{IdT}}, \boldsymbol{\theta}_{\mathrm{Te}}, \boldsymbol{\theta}_{\mathrm{TdI}}, \boldsymbol{\theta}_{\mathrm{TdT}}\right] ;\|\cdot\|_{2}$ 表示 $L_{2}$ 范数; $l_{\mathrm{I}}\left(\boldsymbol{i}_{p}\right.$, $\left.\boldsymbol{t}_{p} ; \boldsymbol{W}_{\mathrm{rec}}\right)$ 和 $l_{\mathrm{T}}\left(\boldsymbol{i}_{p}, \boldsymbol{t}_{p} ; \boldsymbol{W}_{\mathrm{rec}}\right)$ 分别表示图像全模态自 编码器的重构损失函数和文本全模态自编码的重 构损失函数, 定义为

$$
\begin{aligned}
& l_{\mathrm{I}}\left(\boldsymbol{i}_{p}, \boldsymbol{t}_{p} ; \boldsymbol{W}_{\text {rec }}\right)=\left\|\boldsymbol{i}_{p}-\boldsymbol{s}_{p}^{\mathrm{I}}\right\|_{2}^{2}+\left\|\boldsymbol{t}_{p}-\boldsymbol{f}_{p}^{\mathrm{T}}\right\|_{2}^{2} ; \\
& l_{\mathrm{T}}\left(\boldsymbol{i}_{p}, \boldsymbol{t}_{p} ; \boldsymbol{W}_{\text {rec }}\right)=\left\|\boldsymbol{t}_{p}-\boldsymbol{s}_{p}^{\mathrm{T}}\right\|_{2}^{2}+\left\|\boldsymbol{i}_{p}-\boldsymbol{f}_{p}^{\mathrm{I}}\right\|_{2}^{2} .
\end{aligned}
$$

通过最小化 $L_{\mathrm{rec}}\left(\boldsymbol{W}_{\mathrm{rec}}\right)$ 训练网络, 可以减少重构过 程中的信息损失.

为了使不同模态的样本经过编码器嵌人公共 空间中仍然保持语义判别性, 即相同类别的样本 尽可能相似, 不同类别的样本尽可能不相似, 本文 设计了一个分类器 $C$, 将公共空间中不同模态的样 本的嵌人特征映射到类别空间中. 定义语义判别 性损失函数为

$$
\begin{gathered}
L_{\text {dis }}\left(\boldsymbol{W}_{\text {dis }}\right)=\frac{1}{n_{\text {train }}}\left\|\boldsymbol{W}_{\text {dis }} \boldsymbol{H}^{\mathrm{I}}-\boldsymbol{Y}\right\|_{\mathrm{F}}+ \\
\frac{1}{n_{\text {train }}}\left\|\boldsymbol{W}_{\text {dis }} \boldsymbol{H}^{\mathrm{T}}-\boldsymbol{Y}\right\|_{\mathrm{F}} .
\end{gathered}
$$

其中, $\boldsymbol{W}_{\mathrm{dis}}$ 为 $C$ 的参数; \|\|$_{\mathrm{F}}$ 为弗罗贝尼乌斯范数; $\boldsymbol{H}^{\mathrm{I}}=\left[\boldsymbol{h}_{1}^{\mathrm{I}}, \boldsymbol{h}_{2}^{\mathrm{I}}, \cdots, \boldsymbol{h}_{n_{\text {train }}}^{\mathrm{I}}\right]$ 为训练集中图像模态的样本 的嵌人特征矩阵; $\boldsymbol{H}^{\mathrm{T}}=\left[\boldsymbol{h}_{1}^{\mathrm{T}}, \boldsymbol{h}_{2}^{\mathrm{T}}, \cdots, \boldsymbol{h}_{n_{\operatorname{man}}}^{\mathrm{T}}\right]$ 为训练集
中文本模态的样本的嵌人特征矩阵; $\boldsymbol{Y}=$ $\left[\boldsymbol{y}_{1}, \boldsymbol{y}_{2}, \cdots, \boldsymbol{y}_{n_{\text {train }}}\right]$ 为训练样本对的类别标签矩阵. 通 过最小化 $L_{\mathrm{dis}}\left(\boldsymbol{W}_{\mathrm{dis}}\right)$ 训练分类器, 可以使学习到的 嵌人特征具有类别标签中包含的语义判别信息.

不同模态中的成对样本在公共空间中的嵌人 特征应该是相似的，即它们在公共空间中的距离 应该尽可能地小. 定义语义一致性损失函数为

$$
L_{\text {inv }}\left(\boldsymbol{W}_{\text {inv }}\right)=\frac{1}{n_{\text {train }}}\left\|\boldsymbol{H}^{\mathrm{I}}-\boldsymbol{H}^{\mathrm{T}}\right\|_{\mathrm{F}} .
$$

其中, $\boldsymbol{W}_{\mathrm{inv}}=\left[\boldsymbol{\theta}_{\mathrm{Ie}}, \boldsymbol{\theta}_{\mathrm{Te}}\right]$ 为 $G_{\mathrm{Ie}}$ 和 $G_{\mathrm{Te}}$ 的参数集合. 通 过最小化 $L_{\mathrm{inv}}\left(\boldsymbol{W}_{\mathrm{inv}}\right)$, 不同模态中的成对样本在公 共空间的嵌人特征被尽可能地对齐, 在一定程度 上减小了不同模态成对样本之间存在的差异.

综上所述, 定义生成模型损失函数为

$$
\begin{gathered}
L_{\mathrm{gen}}\left(\boldsymbol{W}_{\text {rec }}, \boldsymbol{W}_{\mathrm{dis}}, \boldsymbol{W}_{\mathrm{inv}}\right)=L_{\mathrm{dis}}\left(\boldsymbol{W}_{\mathrm{dis}}\right)+ \\
\alpha \cdot L_{\text {rec }}\left(\boldsymbol{W}_{\text {rec }}\right)+\beta \cdot L_{\text {inv }}\left(\boldsymbol{W}_{\text {inv }}\right)
\end{gathered}
$$

其中， $\alpha$ 和 $\beta$ 用来权衡生成模型损失函数中各项 的重要程度.

\section{4 判别模型}

为了生成具有模态间不变性的特征表示, 本 文引人 $D_{\mathrm{C}}$. 由于一个判别器不能充分地挖掘模态 间的不变性，因此，本文在此基础上新增了 2 个判 别器 $D_{\mathrm{I}}$ 和 $D_{\mathrm{T}}$, 进一步地挖掘模态间的不变性, 使 公共空间能够获得更好的模态间的不变性.

在训练阶段，把图像在公共空间中的嵌人特 征 $\boldsymbol{h}_{p}^{\mathrm{I}}$ 当作正例, 把文本在公共空间中的嵌入特征 $\boldsymbol{h}_{p}^{\mathrm{T}}$ 当作负例, 将它们输人 $D_{\mathrm{C}}$ 中进行对抗训练, 用 $D_{\mathrm{C}}$ 判断它们属于哪个模态. 在 $D_{\mathrm{C}}$ 上定义的对抗 损失函数为

$$
\begin{aligned}
& L_{\text {adv1 }}\left(\boldsymbol{W}_{D_{\mathrm{C}}}\right)=-\frac{1}{n_{\text {train }}} \sum_{p=1}^{n_{\text {train }}}\left(\log D_{\mathrm{C}}\left(\boldsymbol{h}_{p}^{\mathrm{I}} ; \boldsymbol{W}_{D_{\mathrm{C}}}\right)+\right. \\
& \left.\log \left(1-D_{\mathrm{C}}\left(\boldsymbol{h}_{p}^{\mathrm{T}} ; \boldsymbol{W}_{D_{\mathrm{C}}}\right)\right)\right) .
\end{aligned}
$$

其中, $\boldsymbol{W}_{D_{\mathrm{C}}}$ 为 $D_{\mathrm{C}}$ 的参数.

在训练过程中, 将 $G_{\text {IdI }}$ 重构出的 $\boldsymbol{s}_{p}^{\mathrm{I}}$ 当作正例, 将 $G_{\mathrm{TdI}}$ 重构出的 $\boldsymbol{f}_{p}^{\mathrm{I}}$ 当作负例, 将它们输人 $D_{\mathrm{I}}$ 中进 行对抗训练, 训练 $D_{\mathrm{I}}$ 判断重构出的图像特征是来 自图像模态还是文本模态. 在 $D_{\mathrm{I}}$ 上定义的对抗损 失函数为

$$
\begin{gathered}
L_{\mathrm{adv} 2}\left(\boldsymbol{W}_{D_{\mathrm{I}}}\right)=-\frac{1}{n_{\text {train }}} \sum_{p=1}^{n_{\text {train }}}\left(\ln D_{\mathrm{I}}\left(\boldsymbol{s}_{p}^{\mathrm{I}} ; \boldsymbol{W}_{D_{\mathrm{I}}}\right)+\right. \\
\left.\ln \left(1-D_{\mathrm{I}}\left(\boldsymbol{f}_{p}^{\mathrm{I}} ; \boldsymbol{W}_{D_{\mathrm{I}}}\right)\right)\right) .
\end{gathered}
$$

其中, $\boldsymbol{W}_{D_{\mathrm{I}}}$ 为 $D_{\mathrm{I}}$ 的参数. 
类似地, 将 $G_{\mathrm{TdT}}$ 重构出的 $\boldsymbol{s}_{p}^{\mathrm{T}}$ 当作正例, 将 $G_{\mathrm{IdT}}$ 重构出的 $\boldsymbol{f}_{p}^{\mathrm{T}}$ 当作负例, 将它们输人 $D_{\mathrm{T}}$ 进行 对抗训练, 训练 $D_{\mathrm{T}}$ 判断重构出的文本特征是来自 图像模态还是文本模态. 在 $D_{\mathrm{T}}$ 上定义的对抗损失 函数为

$$
\begin{gathered}
L_{\mathrm{adv} 3}\left(\boldsymbol{W}_{D_{\mathrm{T}}}\right)=-\frac{1}{n_{\text {train }}} \sum_{p=1}^{n_{\text {train }}}\left(\ln D_{\mathrm{T}}\left(\boldsymbol{s}_{p}^{\mathrm{T}} ; \boldsymbol{W}_{D_{\mathrm{T}}}\right)+\right. \\
\ln \left(1-D_{\mathrm{T}}\left(\boldsymbol{f}_{p}^{\mathrm{T}} ; \boldsymbol{W}_{D_{\mathrm{T}}}\right)\right) .
\end{gathered}
$$

其中, $\boldsymbol{W}_{D_{\mathrm{T}}}$ 为 $D_{\mathrm{T}}$ 的参数.

综上所述, 定义判别模型损失函数为

$$
\begin{gathered}
L_{\mathrm{adv}}\left(\boldsymbol{W}_{D_{\mathrm{C}}}, \boldsymbol{W}_{D_{\mathrm{I}}}, \boldsymbol{W}_{D_{\mathrm{T}}}\right)=L_{\mathrm{adv} 1}\left(\boldsymbol{W}_{D_{\mathrm{C}}}\right)+ \\
L_{\mathrm{adv} 2}\left(\boldsymbol{W}_{D_{\mathrm{I}}}\right)+L_{\mathrm{adv} 3}\left(\boldsymbol{W}_{D_{\mathrm{T}}}\right) .
\end{gathered}
$$

\section{5 优化过程}

生成模型和判别模型具有相反的优化目标, 它们以对抗的方式进行迭代训练, 每次迭代包括 以下 2 个子过程.

(1) 固定生成模型, 优化判别模型, 目标函数为

$$
\begin{aligned}
& \left(\hat{\boldsymbol{W}}_{D_{\mathrm{C}}}, \hat{\boldsymbol{W}}_{D_{\mathrm{l}}}, \hat{\boldsymbol{W}}_{D_{\mathrm{T}}}\right)=\underset{\boldsymbol{W}_{D_{\mathrm{C}}}, \boldsymbol{W}_{D_{1}}, \boldsymbol{W}_{D_{\mathrm{T}}}}{\operatorname{argmax}}\left(L_{\mathrm{gen}}\left(\hat{\boldsymbol{W}}_{\mathrm{rec}}, \hat{\boldsymbol{W}}_{\mathrm{dis}}, \hat{\boldsymbol{W}}_{\mathrm{inv}}\right)-\right. \\
& \left.L_{\mathrm{adv}}\left(\boldsymbol{W}_{D_{\mathrm{C}}}, \boldsymbol{W}_{D_{\mathrm{l}}}, \boldsymbol{W}_{D_{\mathrm{T}}}\right)\right) .
\end{aligned}
$$

判别模型中各个参数的更新公式分别为

$$
\begin{gathered}
\boldsymbol{W}_{D_{\mathrm{C}}} \leftarrow \boldsymbol{W}_{D_{\mathrm{C}}}+\mu \cdot \nabla_{\boldsymbol{W}_{D_{\mathrm{C}}}} \frac{1}{m}\left(L_{\mathrm{gen}}-L_{\mathrm{adv}}\right), \\
\boldsymbol{W}_{D_{\mathrm{I}}} \leftarrow \boldsymbol{W}_{D_{\mathrm{I}}}+\mu \cdot \nabla_{\boldsymbol{W}_{D_{\mathrm{I}}}} \frac{1}{m}\left(L_{\mathrm{gen}}-L_{\mathrm{adv}}\right), \\
\boldsymbol{W}_{D_{\mathrm{T}}} \leftarrow \boldsymbol{W}_{D_{\mathrm{T}}}+\mu \cdot \nabla_{\boldsymbol{W}_{D_{\mathrm{T}}}} \frac{1}{m}\left(L_{\mathrm{gen}}-L_{\mathrm{adv}}\right) .
\end{gathered}
$$

其中, $\mu$ 为学习率的大小; $m$ 为训练批次的大小.

（2）固定判别模型, 优化生成模型, 目标函数为

$$
\begin{aligned}
& \left(\hat{\boldsymbol{W}}_{\mathrm{rec}}, \hat{\boldsymbol{W}}_{\mathrm{dis}}, \hat{\boldsymbol{W}}_{\mathrm{inv}}\right)=\underset{\boldsymbol{W}_{\text {rec }}, \boldsymbol{W}_{\mathrm{dis}}, \boldsymbol{W}_{\mathrm{inv}}}{\arg \min }\left(L_{\mathrm{gen}}\left(\boldsymbol{W}_{\mathrm{rec}}, \boldsymbol{W}_{\mathrm{dis}}, \boldsymbol{W}_{\mathrm{inv}}\right)-\right. \\
& \left.L_{\mathrm{adv}}\left(\hat{\boldsymbol{W}}_{D_{\mathrm{C}}}, \hat{\boldsymbol{W}}_{D_{\mathrm{I}}}, \hat{\boldsymbol{W}}_{D_{\mathrm{T}}}\right)\right) .
\end{aligned}
$$

生成模型中各个参数的更新公式分别为

$$
\begin{gathered}
\boldsymbol{W}_{\mathrm{rec}} \leftarrow \boldsymbol{W}_{\mathrm{rec}}-\mu \cdot \nabla_{\boldsymbol{W}_{\mathrm{rec}}} \frac{1}{m}\left(L_{\mathrm{gen}}-L_{\mathrm{adv}}\right), \\
\boldsymbol{W}_{\mathrm{dis}} \leftarrow \boldsymbol{W}_{\mathrm{dis}}-\mu \cdot \nabla_{\boldsymbol{W}_{\mathrm{dis}}} \frac{1}{m}\left(L_{\mathrm{gen}}-L_{\mathrm{adv}}\right), \\
\boldsymbol{W}_{\mathrm{inv}} \leftarrow \boldsymbol{W}_{\mathrm{inv}}-\mu \cdot \nabla_{\boldsymbol{W}_{\mathrm{inv}}} \frac{1}{m}\left(L_{\mathrm{gen}}-L_{\mathrm{adv}}\right) .
\end{gathered}
$$

\section{3 实验结果与分析}

\section{1 数据集与评价指标}

本文使用了 3 个在跨模态检索中常用的数据 集 Pascal Sentence, Wikipedia 和 NUS-WIDE-10k; 它们的样本示例如表 1 所示.

Pascal Sentence 数据集是 Pascal VOC 数据集 的子集, 包含 20 个类别, 每个类别有 50 幅图像, 共有 1000 幅图像. 每幅图像与其对应的 5 个句子 组成一个数据对. 本文从每个类别中随机选取 40 个数据对作为训练集, 共有 800 个数据对, 将剩余 的 200 个数据对作为测试集.

Wikipedia 数据集包含 10 个类别, 共有 2866 幅图像, 每幅图像与其对应的文章组成一个数据 对. 本文随机选择 2173 个数据对作为训练集, 将 剩余的 693 个数据对作为测试集.

NUS-WIDE-10k 是 NUS-WIDE 数据集的子集. NUS-WIDE 数据集是一个多标签的数据集, 包含 81 个类别, 共有 269648 幅图像, 每幅图像与其对

表 13 个数据集的样本实例表

\begin{tabular}{ll} 
数据集 & \multicolumn{1}{c|}{ 1. A blue grounded fighter jet is parked on grass in front of a glass building. } \\
2. A blue jet stopped on a lawn. \\
3. A fighter jet sits on display. \\
4. A jet plane on exhibit in front of a modern building. \\
5. Blue fighter jet parked in the green grass.
\end{tabular}


应的文本标注组成一个数据对. 该数据集的类别 非常不平衡，本文使用了其子集 NUS-WIDE-10k, 它由 NUS-WIDE 中数量最多的前 10 个类别中的单 标签样本构成. 本文从每个类别中随机选取 1000 个数据对作为训练集, 共有 8000 个数据对, 将剩 余的 2000 个数据对作为测试集. 表 2 列出了 3 个 数据集的统计信息.

表 23 个数据集的统计信息

\begin{tabular}{lrrccc}
\hline \multicolumn{1}{c}{ 数据集 } & $n_{\text {train }}$ & \multicolumn{1}{c}{$n_{\text {test }}$} & $c$ & $d_{\mathrm{I}}$ & $d_{\mathrm{T}}$ \\
\hline Wikipedia & 2173 & 693 & 10 & 4096 & 3000 \\
Pascal Sentence & 800 & 200 & 20 & 4096 & 1000 \\
NUS-WIDE-10k & 8000 & 2000 & 10 & 4096 & 1000 \\
\hline
\end{tabular}

本文训练网络使用的 $\boldsymbol{i}_{p}$ 由在 ImageNet 数据集 上预训练的 VGGNet-19 提取, $\boldsymbol{t}_{p}$ 由词袋模型(bag of words, BOW)向量表示.

本文使用跨模态检索方法中较常用的指标平 均精度均值(mean average precision, mAP)对检索 的结果进行评估. mAP 同时考虑了跨模态检索的 准确度和检索结果的排序信息. 以 I2T 为例, 用查 询图像 $\boldsymbol{i}_{p}$ 检索相似文本的平均精度 (average precision, $\mathrm{AP})$, 即 $\mathrm{AP}=\frac{1}{L} \sum_{k=1}^{n_{\text {ess }}} \frac{R_{k}}{k} \sigma(k)$. 其中, $L$ 为测试集中 与 $\boldsymbol{i}_{p}$ 相似的文本个数; $R_{k}$ 为前 $k$ 个返回的文本中与 $\boldsymbol{i}_{p}$ 相似的文本个数; $\sigma(k)$ 是一个指示函数, 如果第 $k$ 个返回的文本与 $\boldsymbol{i}_{p}$ 相似，则 $\sigma(k)=1$, 反之 $\sigma(k)=0$. 定义 $\mathrm{mAP}=\frac{1}{n_{\text {test }}} \sum_{k=1}^{n_{\text {test }}} \mathrm{AP}$.

\section{2 实验细节}

本文方法基于 Torch 框架实现, 学习率设置为 0.0001 , 批次大小设置为 100 . 生成模型和判别模 型的具体实现细节如下.

\subsection{1 生成模型}

$G_{\mathrm{Ie}}$ 由维度大小分别为 1024 和 100 的 2 个全 连接层组成, $G_{\mathrm{IdI}}$ 由维度大小分别为 1024 和 4096 的 2 个全连接层组成, $G_{\mathrm{IdT}}$ 由维度大小分别为 1024 和 $d_{\mathrm{T}}$ 的 2 个全连接层组成, $G_{\mathrm{Te}}$ 由维度大小 分别为 1024 和 100 的 2 个全连接层组成. $G_{\mathrm{TdI}}$ 和 $G_{\mathrm{TdT}}$ 的网络结构和各个全连接层的维度大小都与 $G_{\text {IdI }}$ 和 $G_{\text {IdT }}$ 对应相同. $C$ 由维度大小为 $c$ 的一个全 连接层组成.

\subsection{2 判别模型}

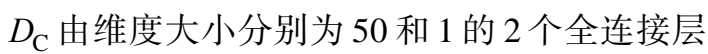

组成, $D_{\mathrm{I}}$ 由维度大小分别为 1024 和 1 的 2 个全连 接层组成, $D_{\mathrm{T}}$ 由维度大小分别为 512 和 1 的 2 个 全连接层组成. 上述所有全连接层的激活函数均 为 ReLU.

\section{3 对比实验}

为了验证本文方法的有效性，选取 10 个主流 方法作为对比方法, 其中包括 3 个传统方法 $\mathrm{CCA}^{[1]}, \mathrm{CFA}^{[2]}$ 和学习耦合的特征空间 (learning coupled feature spaces, LCFS) ${ }^{[4]}$, 以及 7 个基于深 度学习的方法: 对应自编码器 (correspondence autoencoder, Corr-AE) ${ }^{[15]}$, 深度语义匹配(deep semantic matching, Deep-SM) ${ }^{[25]}$, 跨媒体多重深度网 络(cross-media multiple deep network, CMDN) ${ }^{[7]}$, 深度监督的跨模态检索 (deep supervised crossmodal retrieval, DSCMR) ${ }^{[11]}$, MHTN ${ }^{[22]}$, ACMR ${ }^{[19]}$ 和 跨模态生成对抗网络 (cross-modal generative adversarial networks, CM-GANs) ${ }^{[21]}$. 在这 7 个基于深 度学习的方法中, MHTN, ACMR 和 CM-GANs 属于 基于 GAN 的方法, Corr-AE, Deep-SM, CMDN 和 DSCMR 属于非基于 GAN 的方法.

(1) $\mathrm{CCA}^{[1]}$. 通过最大化不同模态样本之间的 相关性, 学习映射矩阵, 将不同模态的样本映射到 一个公共空间中。

(2) $\mathrm{CFA}^{[2]}$. 通过最小化不同模态成对样本之 间的弗罗贝尼乌斯范数, 学习线性映射, 将不同模 态的样本映射到一个公共空间中.

(3) $\mathrm{LCFS}^{[4]}$. 将多模态的数据映射到一个公共 空间中并添加了 $L_{21}$ 范数, 选择具有相关性和判别 性的特征。

(4) Corr- $\mathrm{AE}^{[15]}$. 设计了 2 个子网络, 通过将自 编码器的中间层连接在一起, 对跨模态的相关性 和重构误差进行联合建模.

(5) Deep-SM ${ }^{[25]}$. 提出一个深度语义匹配算法, 验证了卷积神经网络提取的视觉特征在跨模态检 索领域中的优越性.

(6) $\mathrm{CMDN}^{[7]}$. 对模态内和模态间的信息进行 联合建模, 获得各个模态数据单独的特征表示, 然 后结合模态内和模态间的特征获得跨模态数据的 共享特征.

(7) $\operatorname{DSCMR}^{[11]}$. 通过最小化标签空间和公共 特征空间的判别损失，监督模型学习具有判别性 的特征表示.

(8) $\mathrm{MHTN}^{[22]}$. 提出一个模态共享的知识迁移 子网络, 将知识从单模态的源域迁移到跨模态的 
目标域中，并采用对抗机制对生成模型和模态判 别模型进行训练.

(9) $\mathrm{ACMR}^{[19]}$. 将模态分类器和特征映射器进 行对抗训练, 学习具有模态间不变性和模态内判 别性的公共特征表示.

(10) CM-GANs ${ }^{[21]}$. 将跨模态卷积自编码器作 为生成模型, 与 2 种判别模型进行对抗训练, 学习 能够保留模态间不变性的特征.

本文方法和对比方法在 3 个数据集上的 $\mathrm{mAP}$ 如表 3 所示, 表中的平均值表示在 I2T 和 T2I 这 2 个跨模态检索任务上 mAP 的平均值, 加粗的数值 为每列的最大值. 从表 3 可以看到, 在 Pascal Sentence 数据集上, 对比方法中平均值最高的是 CM-GANs, 本文方法的平均值比 CM-GANs 提高 了 4.8\%; 在 Wikipedia 数据集上, 对比方法中平均 值最高的是 ACMR, 本文方法的平均值比 ACMR
提高了 $1.4 \%$; 在 NUS-WIDE-10k 数据集上, 对比 方法中平均值最高的是 DSCMR, 本文方法的平均 值比 DSCMR 提高了 $1.1 \%$. 在这 3 个数据集上, 本 文方法的平均值均是最高值, 证明了本文方法的 有效性.

以 Pascal Sentence 数据集为例, 非基于 GAN 的方法中平均值最高的是 DSCMR, 本文方法的平 均值相较于 DSCMR 提高了 $7.3 \%$, 这是由于生成 对抗网络具有强大的学习判别性特征的能力, 可 以使嵌人公共空间的特征更好地保留模态间的不 变性. 基于 GAN 的方法中平均值最高的是 CM-GANs, 本文方法的平均值比 CM-GANs 提高 了 $4.8 \%$, 这是由于本文新增的 2 个判别器可以在 已有的一个判别器的基础上, 更深层地挖掘模态 间的不变性，使生成模型可以生成能够同时具有 语义判别性和较强的模态间不变性的特征.

表 3 各方法在 3 个数据集上的 mAP 对比

\begin{tabular}{|c|c|c|c|c|c|c|c|c|c|}
\hline \multirow{2}{*}{ 方法 } & \multicolumn{3}{|c|}{ Pascal Sentence } & \multicolumn{3}{|c|}{ Wikipedia } & \multicolumn{3}{|c|}{ NUS-WIDE-10k } \\
\hline & I2T & T2I & 平均值 & I2T & T2I & 平均值 & I2T & T2I & 平均值 \\
\hline $\mathrm{CCA}^{[1]}$ & 0.363 & 0.219 & 0.291 & 0.267 & 0.222 & 0.245 & 0.189 & 0.188 & 0.189 \\
\hline $\mathrm{CFA}^{[2]}$ & 0.187 & 0.216 & 0.202 & 0.334 & 0.297 & 0.316 & 0.400 & 0.299 & 0.350 \\
\hline $\mathrm{LCFS}^{[4]}$ & 0.442 & 0.357 & 0.400 & 0.455 & 0.398 & 0.427 & 0.383 & 0.346 & 0.365 \\
\hline Corr-AE ${ }^{[15]}$ & 0.489 & 0.444 & 0.467 & 0.402 & 0.395 & 0.399 & 0.366 & 0.417 & 0.392 \\
\hline Deep-SM ${ }^{[25]}$ & 0.560 & 0.539 & 0.550 & 0.458 & 0.345 & 0.402 & 0.389 & 0.496 & 0.443 \\
\hline $\mathrm{CMDN}^{[7]}$ & 0.534 & 0.534 & 0.534 & 0.488 & 0.427 & 0.458 & 0.492 & 0.515 & 0.504 \\
\hline $\operatorname{DSCMR}^{[11]}$ & 0.586 & 0.573 & 0.579 & 0.521 & 0.478 & 0.499 & 0.552 & 0.542 & 0.547 \\
\hline $\operatorname{MHTN}^{[22]}$ & 0.496 & 0.500 & 0.498 & 0.541 & 0.461 & 0.501 & 0.520 & 0.534 & 0.527 \\
\hline $\mathrm{ACMR}^{[19]}$ & 0.535 & 0.543 & 0.539 & 0.619 & 0.489 & 0.554 & 0.544 & 0.538 & 0.541 \\
\hline CM-GANs ${ }^{[21]}$ & 0.603 & 0.604 & 0.604 & 0.521 & 0.466 & 0.494 & 0.547 & 0.538 & 0.542 \\
\hline 本文 & 0.661 & 0.643 & 0.652 & 0.621 & 0.515 & 0.568 & 0.564 & 0.552 & 0.558 \\
\hline
\end{tabular}

注. 粗体表示每列的最大值.

\section{4 消融实验}

为了分析本文方法中各个模块或损失函数对 实验结果的影响, 设置了 6 组对比实验, 分别移除 本文方法中不同的子模块: 方法 1 表示移除重构损 失函数; 方法 2 表示移除语义判别性损失函数; 方 法 3 表示移除语义一致性损失函数; 方法 4 表示移 除 $D_{\mathrm{C}}$; 方法 5 表示移除 $D_{\mathrm{I}}$ 和 $D_{\mathrm{T}}$; 方法 6 表示移 除 $D_{\mathrm{C}}, D_{\mathrm{I}}$ 和 $D_{\mathrm{T}}$. 在 Pascal Sentence 数据集上的消 融实验结果如表 4 所示.

本文在生成模型上定义了 3 个损失函数，从表 4 可以看到, 与移除重构损失函数前相比, 方法 1 的平均值下降了 $4.7 \%$, 这是因为重构损失函数可 以使网络重构出的特征与其原始输人特征尽可能
相似, 将编码和解码过程中的信息损失降到最低. 与移除语义判别性损失函数前相比, 方法 2 的平均

表 4 本文方法在 Pascal Sentence 数据集上的 移除不同模块的消融实验 $\mathbf{m A P}$

\begin{tabular}{cccc}
\hline 方法 & I2T & T2I & 平均值 \\
\hline 方法 1 & 0.616 & 0.593 & 0.605 \\
方法 2 & 0.529 & 0.523 & 0.526 \\
方法 3 & 0.445 & 0.373 & 0.409 \\
方法 4 & 0.578 & 0.557 & 0.568 \\
方法 5 & 0.620 & 0.610 & 0.615 \\
方法 6 & 0.552 & 0.530 & 0.541 \\
本文 & $\mathbf{0 . 6 6 1}$ & $\mathbf{0 . 6 4 3}$ & $\mathbf{0 . 6 5 2}$ \\
\hline
\end{tabular}

注. 粗体表示每列的最大值. 
值下降了 $12.6 \%$, 这是因为类别标签中的语义判别 信息在训练过程中起到非常重要的作用, 它可以 使相同类别的样本尽可能相似，使不同类别的样 本尽可能不相似. 与移除语义一致性损失函数前 相比, 方法 3 的平均值下降了 $24.3 \%$, 这是因为在 训练过程中最小化语义一致性损失函数可以尽可 能对齐公共空间中成对的图像和文本，减小不同 模态成对样本之间存在的差异.

为了验证 $D_{\mathrm{C}}, D_{\mathrm{I}}$ 和 $D_{\mathrm{T}}$ 在挖掘模态间不变性 的过程中发挥的作用, 设计了方法 4、方法 5 和方 法 6 共 3 组消融实验. 从表 4 可以看出, 与移除 $D_{\mathrm{C}}$ 前相比, 方法 4 的平均值下降了 $8.4 \%$, 验证了现有 工作中使用的判别器确实能够在一定程度上挖掘 模态间的不变性，提高跨模态检索的精度. 与移除 $D_{\mathrm{I}}$ 和 $D_{\mathrm{T}}$ 前相比, 方法 5 的平均值下降了 $3.7 \%$, 说 明在只使用 $D_{\mathrm{C}}$ 的情况下, 无法充分挖掘模态间的 不变性. 从表 4 还可以看到, 方法 4 的平均值比方 法 6 提高了 $2.7 \%$, 说明 $D_{\mathrm{I}}$ 和 $D_{\mathrm{T}}$ 确实有助于学习 模态间的不变性, 进而提高跨模态检索的精度. 方 法 6 的平均值比本文方法下降了 $11.1 \%$, 说明在不 用任何判别器的情况下, 模态间的不变性不能被 挖掘，跨模态检索的精度会受到极大的限制.

\section{5 参数分析}

式(1)中包含 $\alpha$ 和 $\beta$ 这 2 个超参数. 为了分析 $\alpha$ 和 $\beta$ 对模型性能的影响, 本文在 Pascal Sentence 和 Wikipedia 数据集上进行实验, 分别固定其中一个 参数, 调节另一个参数, 观察后者对检索精度的影 响, 实验结果如图 2 所示. $\alpha$ 控制重构损失函数在 生成模型损失函数中的重要性, 从图 2a 可以看到, 随着 $\alpha$ 值的变化, 2 条折线都呈现出先上升后下降 的趋势, 当 $\alpha$ 的值在 0.01 附近时, 在 2 个数据集上 的 mAP 都达到了最大值. 参数 $\beta$ 控制语义一致性 损失函数在生成模型损失函数中的重要性, 从图 $2 \mathrm{~b}$ 可以看到, 2 条折线的变化趋势和图 $2 \mathrm{a}$ 相同, 当 $\beta$ 的值在 0.1 附近时，在 2 个数据集上的 mAP 都达 到了最大值。

\section{6 收敛性分析}

在 Wikipedia 数据集上进行实验, 分析网络的 收敛性. 在训练过程中对生成模型损失函数 $L_{\mathrm{gen}}\left(\boldsymbol{W}_{\mathrm{rec}}, \boldsymbol{W}_{\mathrm{dis}}, \boldsymbol{W}_{\mathrm{inv}}\right)$ 和判别模型损失函数 $L_{\mathrm{adv}}\left(\boldsymbol{W}_{D_{\mathrm{C}}}\right.$, $\left.\boldsymbol{W}_{D_{1}}, \boldsymbol{W}_{D_{\mathrm{T}}}\right)$ 进行联合优化, 图 3 展示了它们在 200 个 epoch 范围内的变化趋势. 从图 3 可以看出, $L_{\mathrm{gen}}\left(\boldsymbol{W}_{\mathrm{rec}}, \boldsymbol{W}_{\mathrm{dis}}, \boldsymbol{W}_{\mathrm{inv}}\right)$ 一开始呈现出下降的变化趋势, 在第 160 个 epoch 附近开始趋于稳定, $L_{\text {adv }}\left(\boldsymbol{W}_{D_{\mathrm{C}}}\right.$,
$\left.\boldsymbol{W}_{D_{\mathrm{l}}}, \boldsymbol{W}_{D_{\mathrm{T}}}\right)$ 一开始也呈现出下降的变化趋势, 下降 幅度比生成模型损失要小，在第 110 个 epoch 附近 开始趋于稳定. 图 3 的结果显示, 本文方法能够很 好地收敛。
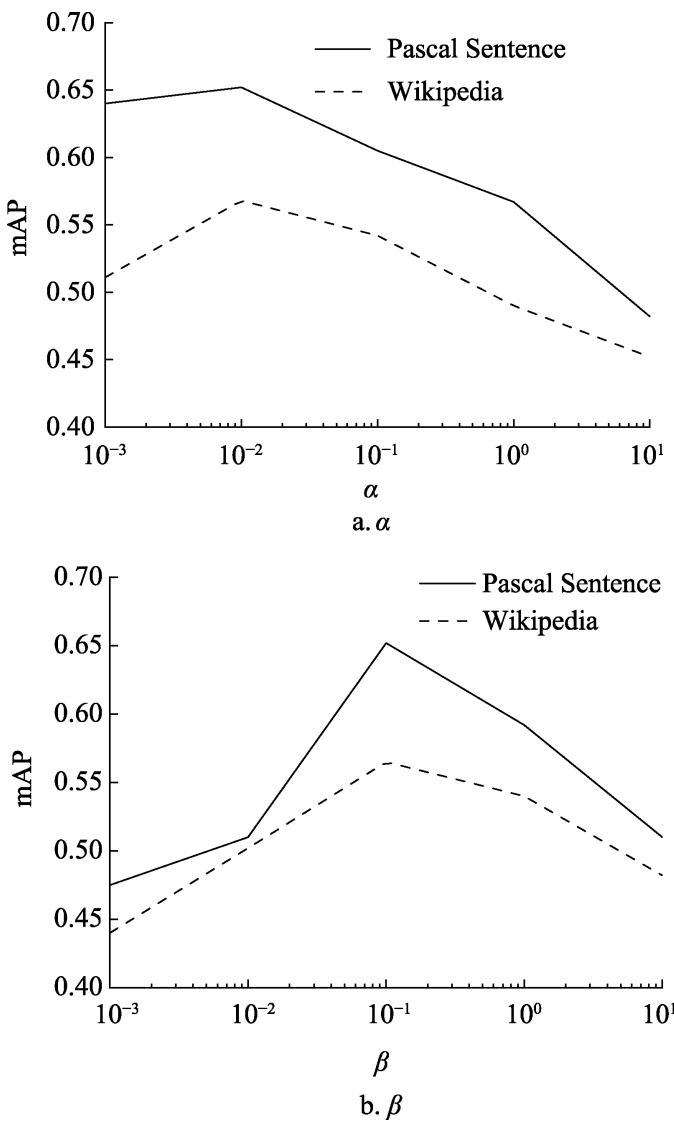

图 2 不同参数值对 $\mathrm{mAP}$ 的影响

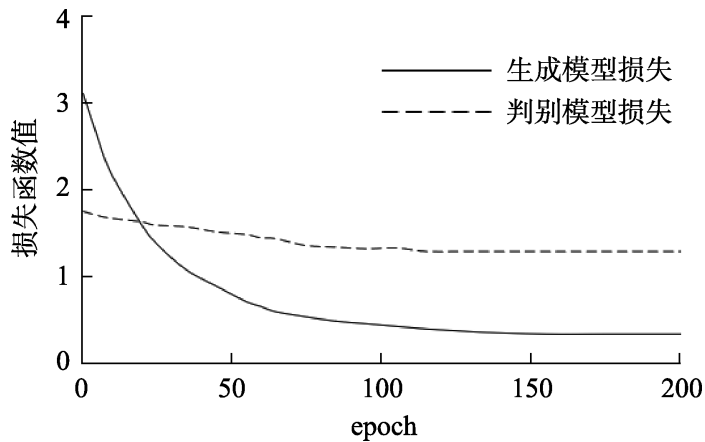

图 3 收敛曲线图

\section{4 结 语}

巨大的模态鸿沟限制了跨模态检索精度的提 升. 为了更好地学习跨模态的公共特征表示, 本文 提出了融合生成对抗机制的全模态自编码器的跨 模检索方法, 将全模态自编码器和分类器作为生 成模型, 通过最小化生成模型损失函数训练生成 
模型, 将不同模态的样本嵌人一个公共空间. 此 外, 还引人一个判别器并在其基础上增加了 2 个判 别器, 与生成模型以对抗的方式进行训练, 使最终 学习到的公共空间不仅保留语义判别性，而且还 保留模态间的不变性. 本文在 3 个跨模态检索领域 常用的数据集上进行实验和分析, 验证了本文方 法的有效性，设置了 6 组消融实验分析本文方法中 各个模块或损失函数对实验结果的影响, 此外, 还 设置了实验对生成模型损失函数中的超参数和网 络的收敛性进行分析.

在未来的工作中，会尝试扩展本文方法，使其 可以面向样本量少、样本标签不足、样本的模态类 型更多等应用场景. 此外, 还可以将本文方法扩展 为学习二进制哈希码的方法, 在提高跨模态检索 的精度的同时，减小存储空间的占用率.

\section{参考文献(References):}

[1] Rasiwasia N, Costa Pereira J, Coviello E, et al. A new approach to cross-modal multimedia retrieval[C] //Proceedings of the 18th ACM International Conference on Multimedia. New York: ACM Press, 2010: 251-260

[2] Li D G, Dimitrova N, Li M K, et al. Multimedia content processing through cross-modal association[C] //Proceedings of the 11th ACM International Conference on Multimedia. New York: ACM Press, 2003: 604-611

[3] Costa Pereira J, Coviello E, Doyle G, et al. On the role of correlation and abstraction in cross-modal multimedia retrieval[J]. IEEE Transactions on Pattern Analysis and Machine Intelligence, 2014, 36(3): 521-535

[4] Wang K Y, He R, Wang W, et al. Learning coupled feature spaces for cross-modal matching[C] //Proceedings of the IEEE International Conference on Computer Vision. Los Alamitos: IEEE Computer Society Press, 2013: 2088-2095

[5] Ranjan V, Rasiwasia N, Jawahar C V. Multi-label cross-modal retrieval[C] //Proceedings of the IEEE International Conference on Computer Vision. Los Alamitos: IEEE Computer Society Press, 2015: 4094-4102

[6] Sharma A, Kumar A, Jacobs D W, et al. Generalized multiview analysis: a discriminative latent space[C] //Proceedings of the IEEE Conference on Computer Vision and Pattern Recognition. Los Alamitos: IEEE Computer Society Press, 2012: 2160-2167

[7] Peng Y X, Huang X, Qi J W. Cross-media shared representation by hierarchical learning with multiple deep networks[C] //Proceedings of the 25th International Joint Conference on Artificial Intelligence. New York: IJCAI Press, 2016: 3846-3853

[8] Huang X, Peng Y X, Yuan M K. Cross-modal common representation learning by hybrid transfer network[C] //Proceedings of the 26th International Joint Conference on Artificial Intelligence. New York: IJCAI Press, 2017: 1893-1900

[9] Peng Y X, Qi J W. Show and tell in the loop: cross-modal circular correlation learning[J]. IEEE Transactions on Multimedia, 2019, 21(6): 1538-1550
[10] Peng Y X, Qi J W, Huang X, et al. CCL: cross-modal correlation learning with multigrained fusion by hierarchical network[J]. IEEE Transactions on Multimedia, 2018, 20(2): 405-420

[11] Zhen L L, Hu P, Wang X, et al. Deep supervised cross-modal retrieval[C] //Proceedings of the IEEE/CVF Conference on Computer Vision and Pattern Recognition. Los Alamitos: IEEE Computer Society Press, 2019: 10386-10395

[12] Hu P, Peng D Z, Zhen L L, et al. Scalable deep multimodal learning for cross-modal retrieval[C] //Proceedings of the 42nd International ACM SIGIR Conference on Research and Development in Information Retrieval. New York: ACM Press, 2019: 635-644

[13] Jiang Q Y, Li W J. Deep cross-modal hashing[C] //Proceedings of the IEEE Conference on Computer Vision and Pattern Recognition. Los Alamitos: IEEE Computer Society Press, 2017: 3270-3278

[14] Yan C, Bai X, Wang S, et al. Cross-modal hashing with semantic deep embedding[J]. Neurocomputing, 2019, 337: 58-66

[15] Feng F X, Wang X J, Li R F. Cross-modal retrieval with correspondence autoencoder[C] //Proceedings of the 22nd ACM International Conference on Multimedia. New York: ACM Press, 2014: 7-16

[16] Ding G G, Guo Y C, Zhou J L, et al. Large-scale cross-modality search via collective matrix factorization hashing[J]. IEEE Transactions on Image Processing, 2016, 25(11): 5427-5440

[17] Yan F, Mikolajczyk K. Deep correlation for matching images and text[C] //Proceedings of the IEEE Computer Society Conference on Computer Vision and Pattern Recognition. Los Alamitos: IEEE Computer Society Press, 2015: 441-450

[18] Su S P, Zhong Z S, Zhang C. Deep joint-semantics reconstructing hashing for large-scale unsupervised cross-modal retrieval[C] //Proceedings of the 17th IEEE/CVF International Conference on Computer Vision. Los Alamitos: IEEE Computer Society Press, 2019: 3027-3035

[19] Wang B K, Yang Y, Xu X, et al. Adversarial cross-modal retrieval[C] //Proceedings of the 25th ACM International Conference on Multimedia. New York: ACM Press, 2017: 154-162

[20] Xu X, He L, Lu H M, et al. Deep adversarial metric learning for cross-modal retrieval[J]. World Wide Web, 2019, 22(2): 657-672

[21] Peng Y X, Qi J W. CM-GANs: cross-modal generative adversarial networks for common representation learning[J]. ACM Transactions on Multimedia Computing, Communications, and Applications, 2019, 15(1): Article No.22

[22] Huang X, Peng Y X, Yuan M K. MHTN: modal-adversarial hybrid transfer network for cross-modal retrieval[J]. IEEE Transactions on Cybernetics, 2020, 50(3): 1047-1059

[23] Wu Y L, Wang S H, Huang Q M. Multi-modal semantic autoencoder for cross-modal retrieval[J]. Neurocomputing, 2019, 331: $165-175$

[24] Xu X, Song J K, Lu H M, et al. Modal-adversarial semantic learning network for extendable cross-modal retrieval[C] //Proceedings of the ACM on International Conference on Multimedia Retrieval. New York: ACM Press, 2018: 46-54

[25] Wei Y C, Zhao Y, Lu C Y, et al. Cross-modal retrieval with CNN visual features: a new baseline[J]. IEEE Transactions on Cybernetics, 2017, 47(2): 449-460 